\title{
Preoperative ALBI grade predicts the outcomes in non-B non-C HCC patients undergoing primary curative resection
}

\section{Yu-Chieh Tsai}

Kaohsiung Chang Gung Memorial Hospital

\section{Fai-Meng Sou}

Kaohsiung Chang Gung Memorial Hospital

\section{Yueh-Wei Liu}

Kaohsiung Chang Gung Memorial Hospital

Chee-Chien Yong

Kaohsiung Chang Gung Memorial Hospital

\section{Pao-Yuan Huang}

Kaohsiung Chang Gung Memorial Hospital

\section{Wei-Ru Cho}

Kaohsiung Chang Gung Memorial Hospital

\section{Tsung-Hui Hu}

Kaohsiung Chang Gung Memorial Hospital

Ming-Chao Tsai ( $\nabla$ tony0779@gmail.com )

Kaohsiung Chang Gung Memorial Hospital

\section{Yi-Ju Wu}

Kaohsiung Chang Gung Memorial Hospital

\section{Ding-Wei Chen}

Kaohsiung Chang Gung Memorial Hospital

Ching-Hui Chuang

Meiho University

Chang-Chun Hsiao

Kaohsiung Chang Gung Memorial Hospital

\section{Research Article}

Keywords: ALBI, NBNC, hepatocellular carcinoma, resection, recurrence

Posted Date: April 8th, 2021

DOI: https://doi.org/10.21203/rs.3.rs-377346/v1 
License: (c) (i) This work is licensed under a Creative Commons Attribution 4.0 International License. Read Full License 
Preoperative ALBI grade predicts the outcomes in non-B non-C HCC patients undergoing primary curative resection

Yu-Chieh Tsai ${ }^{1}$, Fai-Meng Sou ${ }^{1}$, Yueh-Wei Liu ${ }^{2}, Y_{i}-J_{u} \mathrm{Wu}^{2}$, Chee-Chien Yong ${ }^{2}$, Ding-

Wei Chen ${ }^{3}$, Pao-Yuan Huang ${ }^{1}$, Wei-Ru Cho ${ }^{1}$, Ching-Hui Chuang ${ }^{4}$, Chang-Chun

$\mathrm{Hsiao}^{5}$, Tsung-Hui $\mathrm{Hu}^{1}$, Ming-Chao Tsai ${ }^{1,6}$

${ }^{1}$ Division of Hepato-Gastroenterology, Department of Internal Medicine, Kaohsiung Chang Gung Memorial Hospital and Chang Gung University College of Medicine, Kaohsiung, Taiwan; ${ }^{2}$ Liver Transplantation Center and Department of Surgery, Kaohsiung Chang Gung Memorial Hospital and Chang Gung University College of Medicine, Kaohsiung, Taiwan; ${ }^{3}$ Center for Translational Research in Biomedical Sciences, Liver Transplantation Program and Department of Surgery, Kaohsiung Chang Gung Memorial Hospital and Chang Gung University College of Medicine, Kaohsiung, Taiwan; ${ }^{4}$ Department of Nursing, Meiho University, Pingtung, Taiwan; ${ }^{5}$ Graduate Institute of Clinical Medical Sciences, College of Medicine, Chang Gung University, Division of Pulmonary and Critical Care Medicine, Kaohsiung Chang Gung Memorial Hospital; ${ }^{6}$. Graduate Institute of Clinical Medical Sciences, Chang Gung University College of Medicine, Taiwan

\section{Abstract: 248}

Manuscript: 2383

Figures: 3

Tables: 3

\section{Abbreviations:}

NBNC, non-B non-C; HCC, hepatocellular carcinoma; BCLC: Barcelona Clinic Liver 
Cancer; OS: Overall survival; DFS: Disease-free survival; HR: Hazard ratio; HBV: Hepatitis B virus; AFP: alpha-fetoprotein; HBsAg: hepatitis B surface antigen; anti$\mathrm{HBc}$, hepatitis B core antibodies; ALBI, albumin-bilirubin; OBI, occult hepatitis B infection.

\section{Correspondence to}

Ming-Chao Tsai

Division of Hepato-Gastroenterology, Department of Internal Medicine, Kaohsiung Chang Gung Memorial Hospital, 123 Ta Pei Road, Kaohsiung, Taiwan

Tel: +886-7-731-7123, ext. 8310; Fax: +886-7-732-2402;

E-mail: tony0779@gmail.com 


\section{ABSTRACT}

\section{Background}

The albumin-bilirubin (ALBI) grade has been validated as a significant predictor for hepatocellular carcinoma (HCC). However, there is little information about the ALBI grade in patients with non-B non-C HCC (NBNC-HCC) receiving surgery.

\section{Aim}

This study aimed to evaluate ALBI grade as a prognostic factor in patients with NBNC-HCC after primary curative resection.

\section{Method}

This retrospective study enrolled 2137 HCC patients, who received HCC resection between January 2001 and April 2016 at Kaohsiung Chang Gung Memorial Hospital. With exclusion criteria of patients who had chronic hepatitis B or chronic hepatitis C, and prior HCC treatment before resection and received liver transplantation and BCLC stage B or C, finally we enrolled 168 NBNC-HCC patients who received primary curative resection. ALBI score used for grading as well as clinic opathologic features was analyzed, the formula of ALBI score was $\log 10$ [albumin (mg/dL) x 17.1] x 0.66 - albumin (g/dL) x 0.85 .

\section{Result}

There were $66(39.3 \%), 98(58.3 \%)$, and $4(2.4 \%)$ patients who were stratified into ALBI grade I, II, and III, respectively. Patients with ALBI grade II/III had older age $(\mathrm{p}=0.002)$, hypoalbuminemia $(\mathrm{p}<0.001)$, and Child Pugh $\mathrm{B}(\mathrm{p}=0.009)$, they also had poor overall survival compared with those with ALBI grade I $(p=0.003)$. The patients without liver cirrhosis had better survival rate in ALBI grade 1 group $(\mathrm{P}=$ 
0.012). In multivariate analysis, tumor number $(\mathrm{p}=0.001)$ and tumor stages $(\mathrm{pTNM}$ stages $)(p=0.007)$ were independent prognostic factors for recurrence. In predictors for mortality, AFP $(\mathrm{p}=0.004)$, ALBI grade $(\mathrm{p}=0.004)$, tumor number $(\mathrm{P}=0.003)$ and tumor stages ( $\mathrm{pTNM}$ stages) $(\mathrm{p}<0.001)$ were independent prognostic factors.

\section{Conclusion}

Preoperative ALBI grade can be used to predict the mortality in patients with NBNCHCC after primary curative resection.

Keywords: ALBI, NBNC, hepatocellular carcinoma, resection, recurrence 


\section{Introduction}

Hepatocellular carcinoma (HCC) is the fifth most common cancer worldwide,

and is ranked the second most frequent cause of cancer-related death ${ }^{1,2}$. Hepatitis B virus (HBV) and hepatitis $\mathrm{C}$ virus $(\mathrm{HCV})$ infection are the main causes of $\mathrm{HCC}^{3}$. In Taiwan, the major cause of HCC is HBV infection, followed by HCV, similar to many other Asian countries ${ }^{4-6}$. With a universal HBV vaccination program for newborn/infants, the change of lifestyle, and antiviral therapy for HBV and HCV, the incidence of virus-related HCC was decreasing over the last decade. However, the number of HCC patients with neither HBV nor HCV infection, also known as non-B, non-C HCC (NBNC-HCC), has been increasing annually, currently accounting for $11 \%$ of all cases in Taiwan ${ }^{7}$, it is similar with Korea and Japan ${ }^{8,9}$, an HCV-endemic country. These results suggest(showed) that NBNC-HCC is becoming a significant subgroup of HCC in areas of East Asia, and this area was previously endemic for CHB and $\mathrm{CHC}$ and had a high incidence of viral-related HCC.

Curative resection is a potential and the most effective treatment for HCC; it can contribute to survival benefit for patients with early-stage disease when liver transplantation is not immediately accessible ${ }^{10}$, while Hepatic functional reserve has 
always been considered to be critical for outcomes of $\mathrm{HCC}$ patients due to cirrhosis

progression $^{11}$. Child-Pugh grade is the most widely used assessment method for hepatic functional reserve, it includes albumin, PT/INR, ascites and hepatic encephalopathy, but its highly subjective factors, such as severity of ascites and degree of hepatic encephalopathy, may affect assessment ability ${ }^{12,13}$. Recently, there are many studies demonstrated the albumin-bilirubin (ALBI) grade, which was described in 2015 by Johnson et $\mathrm{al}^{14}$, for evaluating hepatic function and predicting the prognosis of patients with HCC following liver resection ${ }^{15-18}$. However, most studies enrolled viral-related $\mathrm{HCC}$, there was little information in regard to the impact of ALBI grade in patients with NBNC-HCC after curative resection. Because the prevalence of nonviral $\mathrm{HCC}$ is increasing in Taiwan, it is a good time to evaluate the effect of the pre-operative ALBI grades in predicting the outcomes of patients with NBNC-HCC after primary curative hepatectomy.

\section{Patients and methods}

\section{Ethics Statement}

This study complies with the standards of the Declaration of Helsinki and current ethical guidelines, and approval was obtained from the Ethics Committee of Chang Gung Memorial Hospital (IRB approval number: 201901103B0). The requirement for informed consent for this study was waived by the Institutional Review Board, and all 
the data were analyzed anonymously.

\section{Patients}

We retrospectively reviewed the Kaohsiung Chang Gung Memorial Hospital HCC registry data, and consisted of data for 2137 HCC patients received HCC resection from January 2001 to April 2016. The HCC diagnosis was based on the criteria of the international guidelines ${ }^{19,20}$ or confirmed by the histology results if those were available. The flow chart of the patients' enrollment is shown in Figure 1. Those who were excluded due to the following reasons: 1134 patients with hepatitis B, 543 patients with hepatitis C, and 97 patients had both hepatitis B and C; 76 patients had been received HCC treatment before resection, 20 patients had received liver transplantation, and 99 patients met BCLC stage C or BCLC stage B with multiple unresectable HCCs tumors; finally, we enrolled 168 NBNC-HCC patients for analysis.

\section{Methods}

Patient's demographics, serum biochemistry, and tumor burden were obtained through medical records review, and diagnosis of cirrhosis was documented by resected non-tumor pathologic report. Blood tests were taken within 1 week before resection. ALBI score was calculated from the formula: ALBI score $=\left(\log _{10}\right.$ bilirubin $\times 0.66)+($ albumin $\times-0.085)$, where the units of bilirubin and albumin were in units of $\mu \mathrm{mol} / 1$ and $\mathrm{g} / \mathrm{l}$, respectively. The ALBI grades were stratified into three grades: grade I, $\leq-2.60$; grade II, -2.60 to $\leq-1.39$; and grade III, $>-1.39$, as reported previously ${ }^{14}$. Disease-free survival (DFS) was defined as the period from tumor 
removal by resection until the detection of recurrence. Overall survival (OS) was defined as the interval between the dates of resection and death, last contact, or 31 December, 2018.

\section{Statistical analysis}

Statistical analyses were performed using SPSS 23.0 statistical package (SPSS,

Inc., Chicago, IL, USA). We used chi-square test and Fisher's exact test for categorial variables. The t-test or Mann-Whitney U test were used for continuous variables. The relationship between DFS, OS, and the ALBI grades were analyzed using KaplanMeier survival curves and the $\log$-rank test, and $\mathrm{p}<0.05$ was considered statistically significant. Factors those were significant in the univariate analysis $(\mathrm{p}<0.05)$ were included in a multivariate analysis by using a Cox forward stepwise variable selection process of the estimated OS and DFS.

\section{Results}

\section{Characteristics of the study population}

Patient characteristics are shown in Table 1. There were $131(78 \%)$ men and 37 $(22 \%)$ women with the mean age of 66 years at enrollment, 58 patients $(34.5 \%)$ had diabetes mellitus. Liver cirrhosis was observed in 38 patients (22.6\%) and high preoperative AFP levels $(>200 \mathrm{ng} / \mathrm{mL})$ were observed in $25(15.3 \%)$ patients. The mean tumor size was $5.3 \pm 3.7 \mathrm{~cm}$, and 4 patients had multiple tumors. As table 1showed, 66, 98, and 4 patients were designated as ALBI grade I, II, and III, respectively; compared to patients with ALBI grade I, patients with ALBI grade II and III were significantly older $(p=0.002)$, lower level of serum albumin $(p<0.001)$, and 
higher percentage of Child-Pugh grade B $(p=0.009)$, but it was no differences in serum bilirubin level and tumor characteristics between these two groups.

\section{Survival analysis}

After a median follow-up of 59 months, 74 patients (44\%) had recurrent HCCs, and $14(8.3 \%)$ died. The one-, three-, and five-year DFS were $80.4 \%, 66.3 \%$, and $56.8 \%$, respectively (Figure 2A). We further investigated the predictive value of the ALBI grade of all subjects, it was no significances between patients with ALBI grades I and II/III (Figure 2B). In the OS analysis, the one-, three-, and five-year OS were $95.8 \%, 86.7 \%$, and $81.9 \%$, respectively (Figure $2 \mathrm{C}$ ) and the groups with ALBI grade I had better OS than patients with ALBI grades II/III $(\mathrm{p}=0.003)$ (Figure 2D). We further stratified by cirrhotic status (Figure 3), NBNC-HCC patients with ALBI grade I had better OS than those with ALBI grades II/III in cirrhotic status $(\mathrm{p}=0.012)$

(Figure 3A), however, there was no significant difference in cirrhotic status (Figure 3B).

\section{Independent factors for DFS and OS of NBNC-HCC patients after curative} resection

Based on multivariate Cox proportion hazards model, AFP $>200 \mathrm{ng} / \mathrm{mL}$ (hazard ration $[\mathrm{HR}], 2.070,95 \% \mathrm{CI}=1.114-3.848, P=0.021)$, tumor number $(\mathrm{HR}, 10.770$, $95 \% \mathrm{CI}=2.513-46.153, P=0.001)$ and $\mathrm{pTNM}$ stages $(\mathrm{HR}, 1.962,95 \% \mathrm{CI}=1.199-$ 3.210, $P=0.007$ ) were independent risk factors for $\mathrm{HCC}$ recurrence (Table 2). In OS analysis, the multivariate Cox proportional hazards model revealed that age $>60$ years $(\mathrm{HR}, 2.939,95 \% \mathrm{CI}=1.995-8.850, P=0.005), \mathrm{AFP}>200 \mathrm{ng} / \mathrm{mL}(\mathrm{HR}, 4.785,95 \%$ $\mathrm{CI}=1.943-11.783, P=0.001)$ ALBI grade II/III $(\mathrm{HR}, 3.689,95 \% \mathrm{CI}=1.512-8.997$, 
$P=0.004)$, multiple tumor number $(\mathrm{HR}, 9.993,95 \% \mathrm{CI}=2.177-45.866, P=0.003)$ and pTNM staging $(\mathrm{HR}, 8.853,95 \% \mathrm{CI}=2.795-28.043, P<0.001)$ were independent risk factors for survival (Table 3), in contrast with those, Child-Pugh grade was not an independent risk factor for survival after adjusting other factors in the multivariate analysis.

\section{Discussion}

To the best of our knowledge, this is the first study to identify pre-operative ALBI grade as a useful biomarker for the assessment of NBNC-HCC after curative resection. OS rates at 5 year post curative resection were $91.8 \%$ in ALBI grade I NBNC-HCC patients, but only $74.8 \%$ in grade II/III NBNC-HCC patients. In the era of preventable strategies for HBV infections and curative intent for HCV infection, the outcomes of NBNC-HCC deserved more attention. Currently, many literatures have been proven that the use of the ALBI score relating to prognosis of post hepatectomy, radiofrequency ablation, transarterial chemoembolization, radiotherapy and systemic therapy ${ }^{21}$. This study was a large-scale cohort study and demonstrated that a higher grade of preoperative ALBI grade correlated with poor OS, but not with HCC recurrence, among NBNC-HCC patients after resection.

The Child-Pugh grading system is traditionally used for liver function 
assessment in patients with liver disease, it was created in the early 1970s as a method for prognostication of chronic liver disease $\mathrm{e}^{22}$. In addition, many HCC staging systems, such as BCLC, Cancer of the Liver Italian Program (CLIP), and Japan integrated staging (JIS), are also integrated Child-Pugh grading. However, ascites and hepatic encephalopathy, two of five parameters for Child-Pugh scoring evaluation, are dependent on physical examination and can be modified by the medication. Recently, the ALBI score has been established for evaluating hepatic functional reserve; in contrast to Child-Pugh scoring, ALBI score only used two objective serological values, albumin and bilirubin, whereas subjective factors as ascites and encephalopathy are not included. A growing number of clinical investigations suggested that ALBI grade can more accurately predict the incidence of postoperative liver failure and OS compared with Child-Pugh system. In the present study, we also compared the areas under the curve (AUC) for the Child-Pugh score and ALBI grade in predicting postoperative survival, and the result showed that ALBI grade had higher AUC compared with Child-Pugh score (0.618 vs. 0.515) (data not shown). This result might be explained that ALBI score classified Child-Pugh grade A into ALBI grade I and grade II with different prognosis at each grade. 
Although HBV and HCV are still the leading causes of HCC, the incidence of NBNC HCC has been increasing in Taiwan ${ }^{19}$; consequently, the rates of resected NBNC HCC in our cohort has also been increasing. The percentage of cases in the present study was increasing, from $13 \%$ in $2001-2005$ to $29 \%$ in $2006-2010$, and finally to $58 \%$ in 2011-2016. The magnificent data implied that NBNC-HCC issue should not be overlooked, although the present study did not have a control arm for patients with viral hepatitis. Comparing to our prior studies ${ }^{17,23}$, patients with NBNCHCC had a higher proportion of diabetes, this result was compatible with a large cohort study in Taiwan, which enrolled 3,843 patients with HCC from The Taiwan Liver Cancer Network (TLCN) ${ }^{7}$. Huang et al has investigated 411 patients with NBNC-HCC, 420 matched patients with HBV-HCC, and 420 matched patients with HCV-HCC, in which the high prevalence (33\%) of DM in the NBNC-HCC cohort. In addition, the degrees of fatty change of liver tissue in our cohort were similar with the above study done by Huang, it was significantly more associated with patients with NBNC-HCC than patients with HBV-HCC or HCV-HCC. Our results confirmed that metabolic risk factors were associated with patients with NBNC-HCC. 
In the present study, aside from the ALBI grade, we also found that serum AFP, multiple tumor number, and pTNM stage were independent risk factors for HCC recurrence. Furthermore, age, AFP, tumor number and pTNM stage were risk factors for OS. These results were similar with the previous studies ${ }^{17,24,25}$, but not all the same, the difference from the previous studies may be due to the HCC population. In the present study, we focused on the NBNC-HCC patients after curative resection, while the previous studies focusing on the HBV or HCV or both related HCC. The underlying mechanism of HCC from non-viral hepatitis must (may) be different from viral hepatitis. However, we believed that preoperative ALBI grade is a useful marker for predicting the outcomes of $\mathrm{HCC}$ patients after curative resection regardless of patients with HBV-, HCV-, or NBNC-related HCC.

In the present study, we did not have data regarding the occult hepatitis B infection $(\mathrm{OBI})$, and it was reported that $\mathrm{OBI}$ played an important role in the progression of cirrhosis and the development of HCC in several epidemiological and molecular studies. OBI is defined as the presence of replication-competent HBV DNA in the liver and/or HBV DNA in the blood in person with serum HBsAg negativity assessed by currently available assays ${ }^{26}$. OBI is the combined result of host immune 
control and different genomic expressions of the virus, it leads to a virological quiescent state, hence, the vast majority of OBI cases have low levels of HBV DNA ${ }^{27}$. The prevalence of OBI varied from region to region worldwide and patient populations, a higher rates reported in $\mathrm{Asia}^{28}$. In Taiwan, the prevalence was $0.11 \%$ in blood donors ${ }^{29}$ and $10.9 \%$ in $\mathrm{HBV}$ vaccinated children ${ }^{30}$, and in patients with HBsAgnegative HCC, the prevalence of OBI may be higher. A meta-analysis showed an increased risk of $\mathrm{HCC}$ in both retrospective (OR: 6.06) and prospective studies (OR: $2.86)^{31}$, although most patients did not have the data of serum HBV DNA for evaluation whether OBI might be involved, the result of ALBI grade predicting the outcomes of NBNC-HCC after resection was unchanged.

We acknowledge the following limitations. First, this study was a single-center retrospective study, in this study, we didn't collect the data by the intraoperative blood loss, amount of fluid received, blood transfusion, and the size of the remnant liver volume, but previous study showed that the above data were not statistically significant ${ }^{32}$. Second, this was a retrospective data from medical record and some patient were lost follow up; therefore, for prognosis evaluation, a prospective study is needed for further assessment on the precise time of ALBI grade. Moreover, in the 
present study, due to the missing data, we could not collect the data of hepatitis core antibodies (anti-HBc) to exclude possible occult or past HBV infections among patients with NBNC-HCC despite HBsAg-negative, especially in an $\mathrm{HBV}$-endemic country. The complete analysis including the status of anti-HBc, hepatitis B surface antibody, and HBV DNA, was our noteworthy strengths in this study to clarify the role of occult HBV infection in these patients. In the present study, we excluded the data of hepatitis B core antibodies (anti-HBc) for no related data in the medical records of the patients with HCC. Occult hepatitis viral infection may also require to considerate that Taiwan is an endemic area for HBV infection, and the prevalence of anti-HBc may be high for those born before universal vaccination was instituted.

\section{Conclusions}

In conclusion, we concluded that ALBI grade may predict the OS in NBNC-HCC patients after curative resection. On the other hand, although there was no significant difference in HCC recurrence stratified by ALBI grade, we still observed that patients with ALBI grade II/III had poorer DFS than those with ALBI grade I. Hence, we believed that the ALBI grade is a promising noninvasive marker for prediction the outcomes of NBNC-related HCC patients after curative resection. 


\section{Declarations}

\section{Ethics approval and consent to participate}

The clinical data was acquired with the approval and permission of the Institutional Review Board of the Kaohsiung Chang Gung Memorial Hospital. The study protocol was approved by the Institutional Review Board of the Kaohsiung Chang Gung Memorial Hospital. The written informed consent was waived according to Institutional Review Board due to the retrospective design of the study with no relevant to human biological ethic problems.

\section{Consent for publication}

Not applicable.

\section{Availability of data and material}

All analyzed data are included in this published article. The original data are available upon reasonable request to the corresponding author.

\section{Funding}

This research received no specific grant from any funding agency in the public, commercial, or not-for-profit sectors. This study was supported by grants CMRPG8F0661 and CMRPG890161 from Chang Gung Memorial Hospital, Taiwan. The interpretation and reporting of the data were the sole responsibility of the authors.

\section{Acknowledgments}

This study was supported by grants CMRPG8F0661 and CMRPG890161 from Chang Gung Memorial Hospital, Taiwan. The authors would like to thank all of the patients and their providers who participated in this study. We appreciated the Biostatistics Center, Kaohsiung Chang Gung Memorial Hospital for statistics work.

\section{Author Contributions}

Conception and design: Ming-Chao Tsai; Manuscript writing: Yu-Chieh Tsai; Collection and assembly of data: Fai-Meng Sou, Yueh-Wei Liu, Yi-Ju Wu, CheeChien Yong, Kuang-Den Chen, Pao-Yuan Huang, Wei-Ru Cho, Ching-Hui Chuang, Chang-Chun Hsiao, Tsung-Hui Hu; Data analysis and interpretation: Ching-Hui 
Chuang, Chang-Chun Hsiao. All authors approved the final version of the manuscript.

Competing Interests: The authors declare no competing interests.

\section{References:}

1. Llovet JM, Zucman-Rossi J, Pikarsky E, et al. Hepatocellular carcinoma. Nat Rev Dis Primers 2016;2:16018.

2. Torre LA, Bray F, Siegel RL, et al. Global cancer statistics, 2012. CA Cancer J Clin 2015;65:87-108.

3. Yang JD, Hainaut P, Gores GJ, et al. A global view of hepatocellular carcinoma: trends, risk, prevention and management. Nat Rev Gastroenterol Hepatol 2019;16:589-604.

4. Liaw YF, Tai DI, Chu CM, et al. Early detection of hepatocellular carcinoma in patients with chronic type B hepatitis. A prospective study.

Gastroenterology 1986;90:263-7.

5. Chen DS. From hepatitis to hepatoma: lessons from type B viral hepatitis. Science 1993;262:369-70.

6. Lu SN, Su WW, Yang SS, et al. Secular trends and geographic variations of hepatitis B virus and hepatitis C virus-associated hepatocellular carcinoma in Taiwan. Int J Cancer 2006;119:1946-52.

7. Huang SF, Chang IC, Hong CC, et al. Metabolic risk factors are associated with non-hepatitis B non-hepatitis $\mathrm{C}$ hepatocellular carcinoma in Taiwan, an endemic area of chronic hepatitis B. Hepatol Commun 2018;2:747-759.

8. Lee SB, Kim KM, An J, et al. Clinical characteristics and potential aetiologies of non-B non-C hepatocellular carcinoma in hepatitis B virus endemic area. Liver Int 2016;36:1351-61.

9. Tateishi R, Uchino K, Fujiwara N, et al. A nationwide survey on non-B, non-C hepatocellular carcinoma in Japan: 2011-2015 update. J Gastroenterol 2019;54:367-376.

10. El-Serag HB. Hepatocellular carcinoma. N Engl J Med 2011;365:1118-27.

11. Durand F, Valla D. Assessment of the prognosis of cirrhosis: Child-Pugh versus MELD. J Hepatol 2005;42 Suppl:S100-7.

12. Fleck A, Raines G, Hawker F, et al. Increased vascular permeability: a major 
cause of hypoalbuminaemia in disease and injury. Lancet 1985;1:781-4.

13. Henriksen JH, Parving HH, Christiansen L, et al. Increased transvascular escape rate of albumin during experimental portal and hepatic venous hypertension in the pig. Relation to findings in patients with cirrhosis of the liver. Scand J Clin Lab Invest 1981;41:289-99.

14. Johnson PJ, Berhane S, Kagebayashi C, et al. Assessment of liver function in patients with hepatocellular carcinoma: a new evidence-based approach-the ALBI grade. J Clin Oncol 2015;33:550-8.

15. Luo HM, Zhao SZ, Li C, et al. Preoperative platelet-albumin-bilirubin grades predict the prognosis of patients with hepatitis B virus-related hepatocellular carcinoma after liver resection: A retrospective study. Medicine (Baltimore) 2018;97:e0226.

16. Liao R, Du CY, Gong JP, et al. HBV-DNA Load-Related Peritumoral Inflammation and ALBI Scores Predict HBV Associated Hepatocellular Carcinoma Prognosis after Curative Resection. J Oncol 2018;2018:9289421.

17. Cho WR, Hung $\mathrm{CH}$, Chen $\mathrm{CH}$, et al. Ability of the post-operative ALBI grade to predict the outcomes of hepatocellular carcinoma after curative surgery. Sci Rep 2020;10:7290.

18. Lin CY, Lin CC, Wang CC, et al. The ALBI Grade is a Good Predictive Model for Very Late Recurrence in Patients with Hepatocellular Carcinoma Undergoing Primary Resection. World J Surg 2020;44:247-257.

19. Bruix J, Sherman M, Practice Guidelines Committee AAftSoLD. Management of hepatocellular carcinoma. Hepatology 2005;42:1208-36.

20. European Association For The Study Of The L, European Organisation For R, Treatment Of C. EASL-EORTC clinical practice guidelines: management of hepatocellular carcinoma. J Hepatol 2012;56:908-43.

21. Deng M, Ng SWY, Cheung ST, et al. Clinical application of AlbuminBilirubin (ALBI) score: The current status. Surgeon 2020;18:178-186.

22. Pugh RN, Murray-Lyon IM, Dawson JL, et al. Transection of the oesophagus for bleeding oesophageal varices. Br J Surg 1973;60:646-9.

23. Huang PY, Wang CC, Lin CC, et al. Predictive Effects of Inflammatory Scores in Patients with BCLC 0-A Hepatocellular Carcinoma after Hepatectomy. J Clin Med 2019;8.

24. Wu JC, Huang YH, Chau GY, et al. Risk factors for early and late recurrence in hepatitis B-related hepatocellular carcinoma. J Hepatol 2009;51:890-7. 
25. Tabrizian P, Jibara G, Shrager B, et al. Recurrence of hepatocellular cancer after resection: patterns, treatments, and prognosis. Ann Surg 2015;261:94755.

26. Raimondo G, Locarnini S, Pollicino T, et al. Update of the statements on biology and clinical impact of occult hepatitis B virus infection. J Hepatol 2019;71:397-408.

27. Makvandi M. Update on occult hepatitis B virus infection. World J Gastroenterol 2016;22:8720-8734.

28. Torbenson M, Thomas DL. Occult hepatitis B. Lancet Infect Dis 2002;2:47986.

29. Su TH, Chen PJ, Chen TC, et al. The clinical significance of occult hepatitis B transfusion in Taiwan--a look-back study. Transfus Med 2011;21:33-41.

30. Mu SC, Lin YM, Jow GM, et al. Occult hepatitis B virus infection in hepatitis B vaccinated children in Taiwan. J Hepatol 2009;50:264-72.

31. Shi Y, Wu YH, Wu W, et al. Association between occult hepatitis B infection and the risk of hepatocellular carcinoma: a meta-analysis. Liver Int 2012;32:231-40.

32. Fagenson AM, Gleeson EM, Pitt HA, et al. Albumin-Bilirubin Score vs Model for End-Stage Liver Disease in Predicting Post-Hepatectomy Outcomes. J Am Coll Surg 2020;230:637-645. 
Figure legends:

Figure 1. Schematic flowchart of the enrolment process

Figure 2. The disease-free survival (DFS) rate and overall survival (OS) rate in NBNC-HCC after resection. (A) DFS in the study population (B) DFS between ALBI Gr I vs. Gr II/III (C) OS in the study population (D) DFS between ALBI Gr I vs. Gr II/III.

Figure 3. The cumulative overall survival in patients with NBNC-related HCC after curative resection among (A) non cirrhotic patients and (B) cirrhotic patients. 
Figure 1. Schematic flowchart of the enrolment process

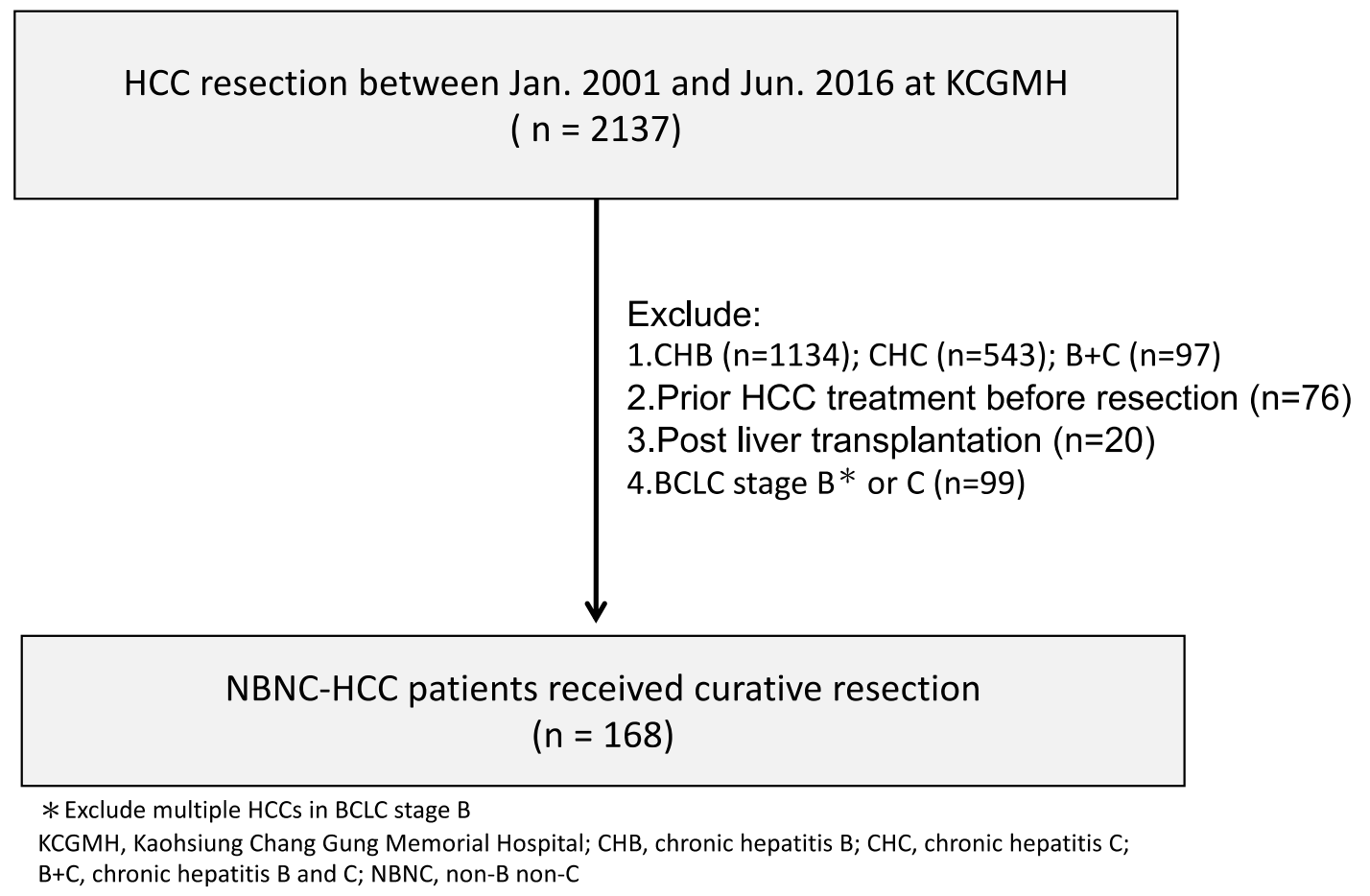


Figure 2. The disease-free survival (DFS) rate and overall survival (OS) rate in NBNC-HCC after resection. (A) DFS in the study population (B) DFS between ALBI Gr I vs. Gr II/III (C) OS in the study population (D) DFS between ALBI Gr I vs. Gr II/III.

(A)

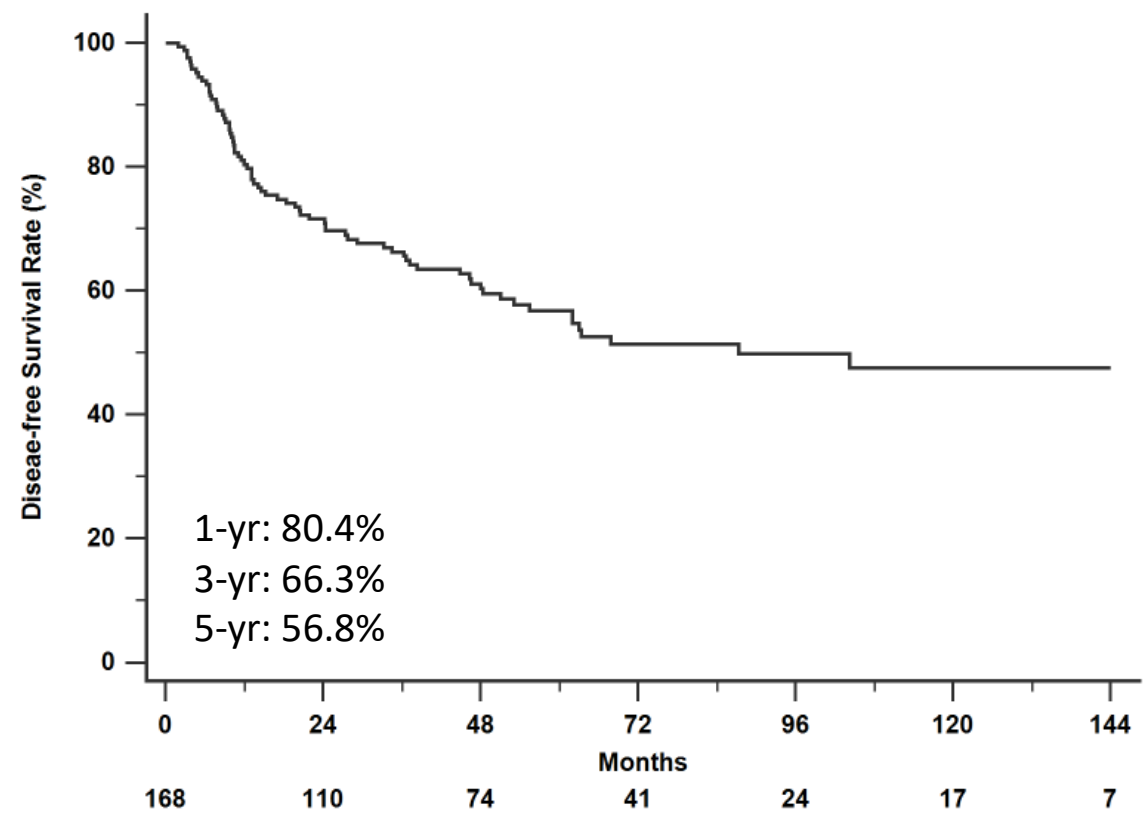

(B)

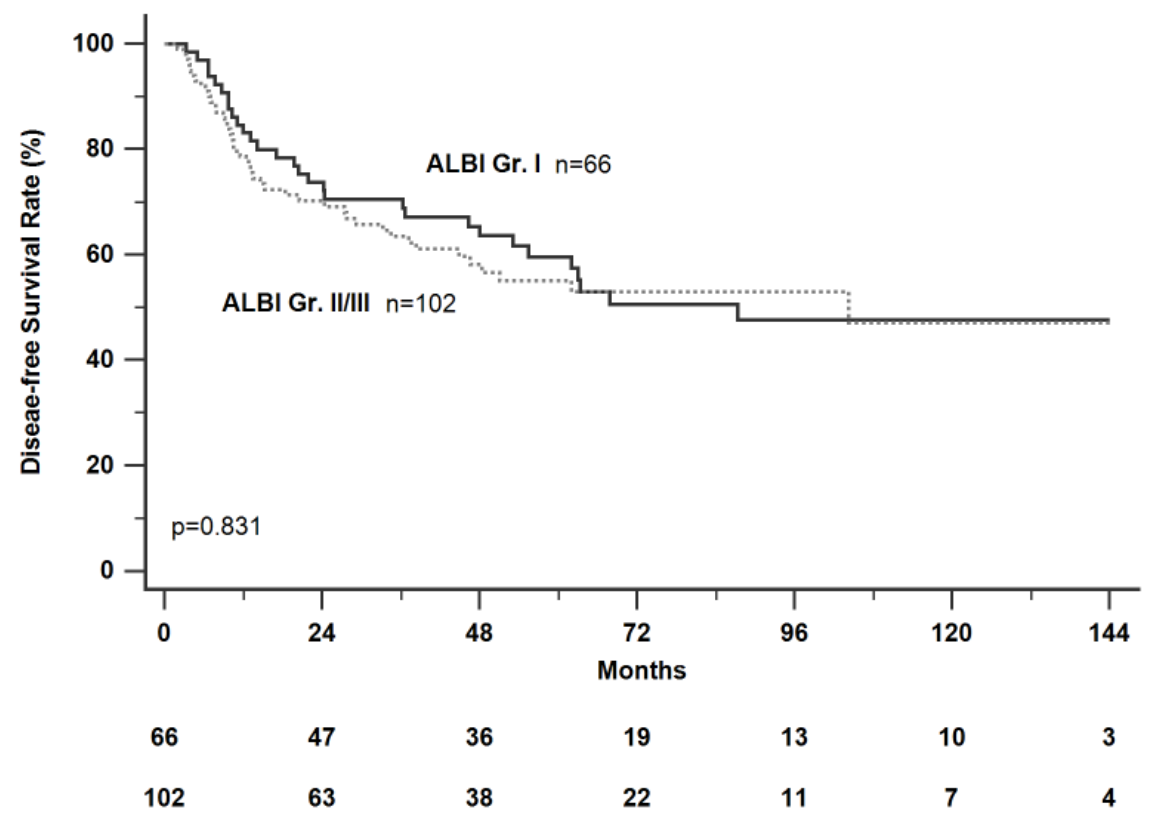


(C)

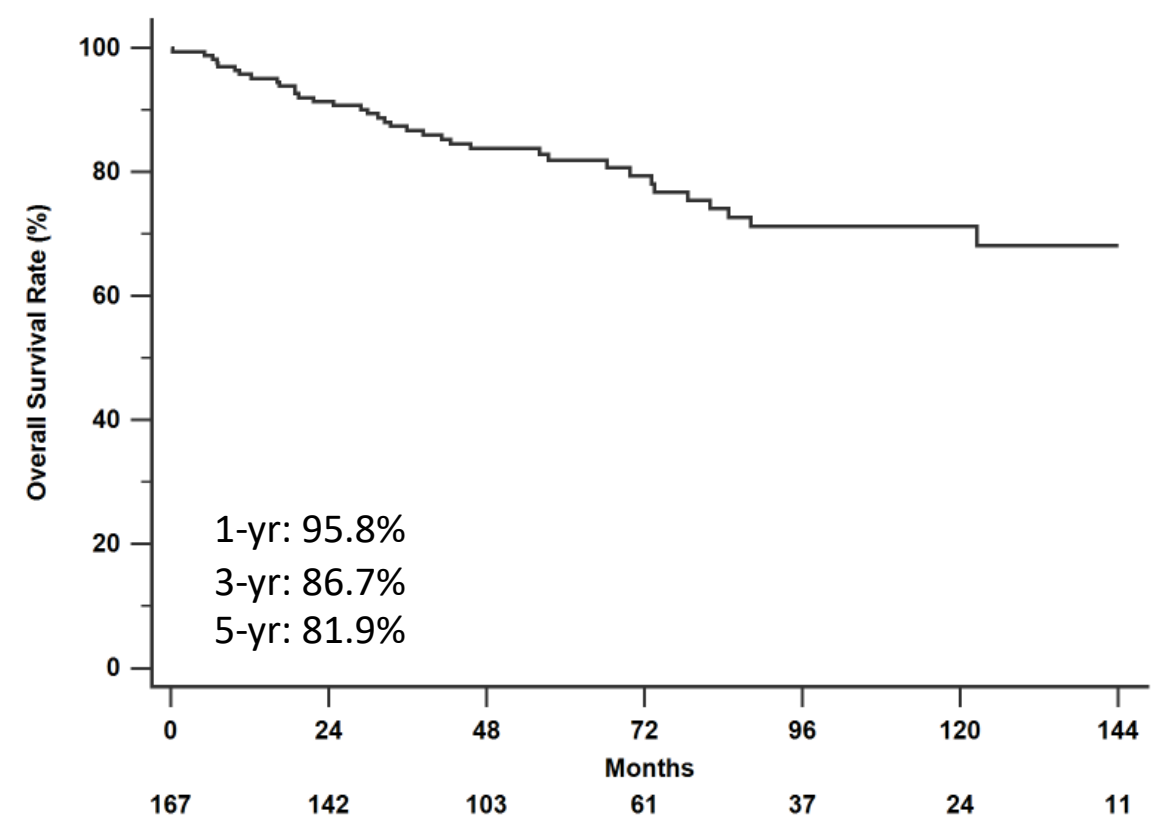

(D)

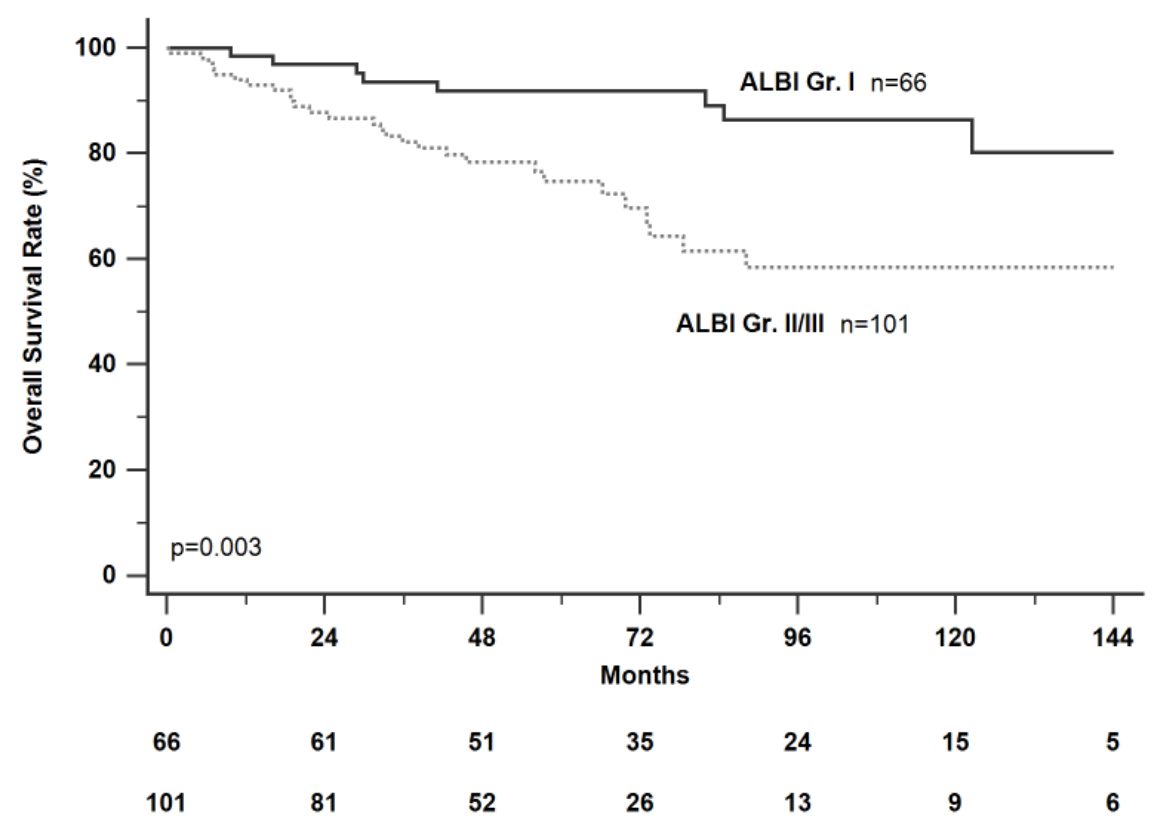


Figure 3. The cumulative overall survival in patients with NBNC-related HCC after curative resection among (A) non cirrhotic patients and (B) cirrhotic patients.

(A)

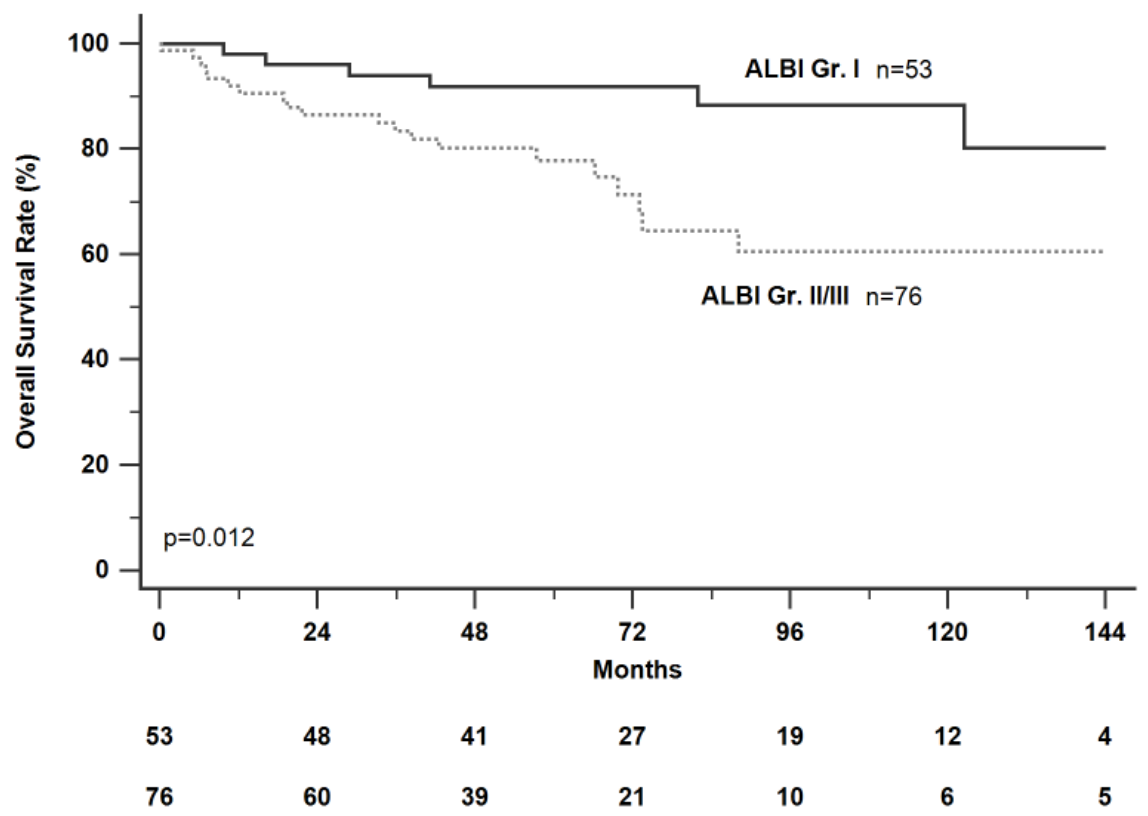

(B)

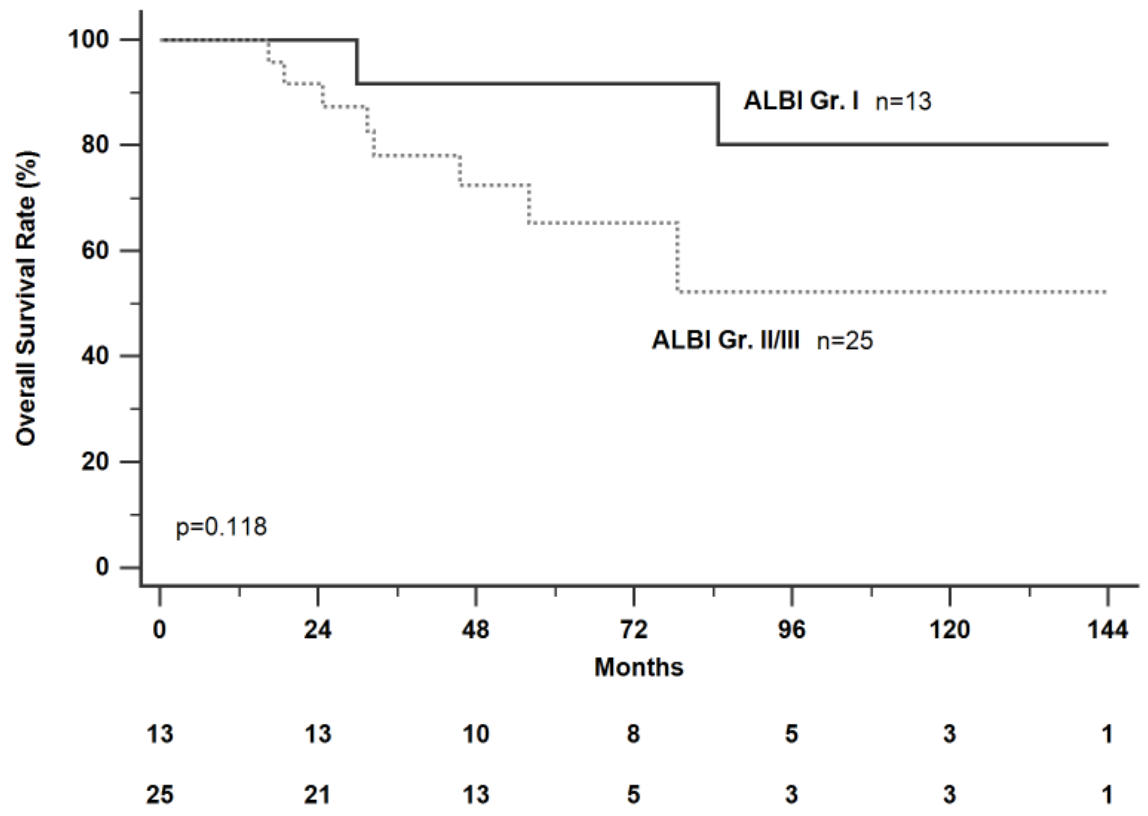


Table 1. Comparison of clinical and pathological characteristics between patients with pre-operative ALBI grades I and II

\begin{tabular}{|c|c|c|c|c|}
\hline & Total $(n=168)$ & ALBI grade $\mathrm{I}(\mathrm{n}=66)$ & ALBI grade II/III $(\mathrm{n}=102)^{*}$ & $P$ value \\
\hline Age (years; mean $\pm \mathrm{SD})$ & $65.2 \pm 12.2$ & $58.9 \pm 11.8$ & $64.8 \pm 12$ & 0.002 \\
\hline Male, n (\%) & $131(78 \%)$ & $56(84.8 \%)$ & $75(73.5 \%)$ & 0.084 \\
\hline Diabetes mellitus, n (\%) & $58(34.5 \%)$ & $22(33.3 \%)$ & $36(35.3 \%)$ & 0.794 \\
\hline Hypertension, n (\%) & $90(58.1 \%)$ & $37(60.7 \%)$ & $53(56.4 \%)$ & 0.598 \\
\hline Current alcohol intake $^{\$}, \mathrm{n}(\%)$ & $32(24.2 \%)$ & $16(32.7 \%)$ & $16(19.3 \%)$ & 0.083 \\
\hline $\operatorname{AST}(\mathrm{U} / \mathrm{L} ;$ mean $\pm \mathrm{SD})$ & $41.6 \pm 74.4$ & $34.7 \pm 19.3$ & $46.1 \pm 94.1$ & 0.333 \\
\hline $\operatorname{ALT}(\mathrm{U} / \mathrm{L} ;$ mean $\pm \mathrm{SD})$ & $44.1 \pm 109.4$ & $39.5 \pm 23.3$ & $47.2 \pm 139.3$ & 0.658 \\
\hline Total bilirubin $(\mathrm{mg} / \mathrm{dL}$; mean $\pm \mathrm{SD})$ & $0.8 \pm 0.3$ & $0.8 \pm 0.3$ & $0.8 \pm 0.4$ & 0.132 \\
\hline $\operatorname{Albumin}(\mathrm{g} / \mathrm{dL} ;$ mean $\pm \mathrm{SD})$ & $3.7 \pm 0.6$ & $4.2 \pm 0.3$ & $3.4 \pm 0.4$ & $<0.001$ \\
\hline Platelet $(<150000 \mathrm{u} / 1), \mathrm{n}(\%)$ & $38(22.9 \%)$ & $14(21.5 \%)$ & $24(23.8 \%)$ & 0.739 \\
\hline $\operatorname{AFP}(>200 \mathrm{ng} / \mathrm{ml}), \mathrm{n}(\%)$ & $25(15.3 \%)$ & $11(16.9 \%)$ & $14(14.3 \%)$ & 0.647 \\
\hline Liver cirrhosis, $\mathrm{n}(\%)$ & $38(22.6 \%)$ & $13(19.7 \%)$ & $25(24.5 \%)$ & 0.466 \\
\hline Tumor size (cm; mean $\pm \mathrm{SD})$ & $5.3 \pm 3.7$ & $4.5 \pm 2.9$ & $5.9 \pm 4.1$ & 0.09 \\
\hline Tumor number (single: multiple) & $164: 4$ & $65: 1$ & $99: 3$ & 0.554 \\
\hline Child-Pugh grade (A : B) & $158: 10$ & $66: 0$ & $92: 10$ & 0.009 \\
\hline BCLC stage $(0: A: B)$ & $17: 87: 64$ & $10: 35: 21$ & $7: 52: 43$ & 0.144 \\
\hline \multicolumn{5}{|l|}{ Pathological features } \\
\hline Fat content $(\%)$ in nontumor tissue, $\mathrm{n}(\%)^{* *}$ & & & & 0.064 \\
\hline
\end{tabular}




\begin{tabular}{|c|c|c|c|c|}
\hline$>30 \%$ & $24(17.5 \%)$ & $15(26.3 \%)$ & $9(11.3 \%)$ & \\
\hline $5 \%-30 \%$ & $37(27.0 \%)$ & $15(26.3 \%)$ & $22(27.5 \%)$ & \\
\hline$<5 \%$ & $76(55.5 \%)$ & $27(47.4 \%)$ & $49(61.3 \%)$ & \\
\hline Vascular invasion, $\mathrm{n}(\%)$ & $55(35 \%)$ & $19(29.7 \%)$ & $36(38.7 \%)$ & 0.224 \\
\hline pTNM stage (I : II : III) & $101: 58: 8$ & $41: 23: 1$ & $60: 35: 7$ & 0.289 \\
\hline Histological grade (well : moderate: poor) & $31: 125: 11$ & $14: 50: 2$ & $17: 75: 9$ & 0.365 \\
\hline
\end{tabular}

$\mathrm{AST}=$ Aspartate aminotransferase; $\mathrm{ALT}=\mathrm{Alanine}$ aminotransferase; $\mathrm{AFP}=\alpha$-fetoprotein; $\mathrm{BCLC}=$ Barcelona $\mathrm{Clinic}$ Liver $\mathrm{Cancer}$; $\mathrm{ALBI}=$ albumin-bilirubin

*4 cases are ALBI grade III

$\$>80$ g ethanol per day

**137 cases had available data 
Table 2. Univariate and multivariate analysis of prognostics factors for recurrence in NBNC-HCC patients after curative resection

\begin{tabular}{|c|c|c|c|c|c|}
\hline \multirow[b]{2}{*}{ Variable } & \multirow[b]{2}{*}{ Comparison } & \multicolumn{2}{|c|}{ Univariate } & \multicolumn{2}{|c|}{ Multivariate } \\
\hline & & HR $(95 \% \mathrm{CI})$ & P value & HR (95\%CI) & P value \\
\hline Age(years) & $>60$ vs $\leqq 60$ & $1.316(0.815-2.125)$ & 0.216 & & \\
\hline Sex & Male vs. Female & $1.220(0.698-2.131)$ & 0.485 & & \\
\hline DM history & Yes vs. No & $1.286(0.800-2.068)$ & 0.299 & & \\
\hline Hypertension & Yes vs. No & $1.184(0.694-2.020)$ & 0.536 & & \\
\hline Alcoholic history & Yes vs. No & $1.353(0.748-2.446)$ & 0.318 & & \\
\hline AFP (ng/ml) & $>200$ vs. $\leqq 200$ & $2.065(1.149-3.710)$ & 0.015 & $2.070(1.114-3.848)$ & 0.021 \\
\hline Platelet $\left(10^{9} / \mathrm{L}\right)$ & $\leqq 150$ vs. $>150$ & $1.264(0.754-2.119)$ & 0.375 & & \\
\hline $\operatorname{Albumin}(\mathrm{g} / \mathrm{dL})$ & $\leqq 3$ vs. $>3$ & $1.358(0.833-2.215)$ & 0.220 & & \\
\hline Liver cirrhosis & Yes vs. No & $0.950(0.552-1.635)$ & 0.853 & & \\
\hline Child-Pugh grade & B vs. A & $1.216(0.682-2.168)$ & 0.508 & & \\
\hline ALBI grade & II/III vs. I & $1.052(0.662-1.671)$ & 0.831 & & \\
\hline Tumor size $(\mathrm{cm})$ & $>5$ vs. $\leqq 5$ & $1.024(0.634-1.653)$ & 0.923 & & \\
\hline Tumor no. & Multiple vs. Single & $2.847(0.892-9.084)$ & 0.077 & $10.770(2.513-46.153)$ & 0.001 \\
\hline BCLC stage & B vs. 0/A & $1.004(0.625-1.615)$ & 0.985 & & \\
\hline Liver fat content $(\%)$ & $>30$ vs. $\leqq 30$ & $1.472(0.811-2.673)$ & 0.204 & & \\
\hline
\end{tabular}




$\begin{array}{lllll}\text { Vascular invasion } & \text { Yes vs. No } & 0.798(0.479-1.329) & 0.386 & \\ \text { pTNM stages } & \text { II+III vs. I } & 2.084(1.302-3.336) & 0.002 & 1.962(1.199-3.210) \\ \text { Histology stages } & \text { Poor vs. well/moderate } & 1.699(0.682-4.232) & 0.255 & \end{array}$

$\mathrm{DM}=$ Diabetes mellitus; $\mathrm{AST}=$ Aspartate aminotransferase; $\mathrm{ALBI}=$ albumin-bilirubin ; $\mathrm{BCLC}=$ Barcelona Clinic Liver Cancer; 
Table 3. Univariate and multivariate analysis of prognostics factors for overall survival in NBNC-HCC patients after curative resection

\begin{tabular}{|c|c|c|c|c|c|}
\hline \multirow[b]{2}{*}{ Variable } & \multirow[b]{2}{*}{ Comparison } & \multicolumn{2}{|c|}{ Univariate } & \multicolumn{2}{|c|}{ Multivariate } \\
\hline & & HR $(95 \% C I)$ & Pvalue & HR $(95 \% C I)$ & P value \\
\hline Age(years) & $>60$ vs $\leqq 60$ & $2.117(0.995-4.507)$ & 0.052 & $2.939(1.995-8.850)$ & 0.005 \\
\hline Sex & Male vs. Female & $0.983(0.462-2.093)$ & 0.965 & & \\
\hline DM & Yes vs. No & $1.324(0.670-2.617)$ & 0.420 & & \\
\hline Hypertension & Yes vs. No & $0.885(0.364-2.152)$ & 0.787 & & \\
\hline Alcoholic history & Yes vs. No & $1.601(0.630-4.064)$ & 0.322 & & \\
\hline AFP (ng/ml) & $>200$ vs. $\leqq 200$ & $3.475(1.648-7.329)$ & 0.001 & $4.785(1.943-11.783)$ & 0.001 \\
\hline Platelet $\left(10^{9} / \mathrm{L}\right)$ & $\leqq 150$ vs. $>150$ & $0.666(0.276-1.605)$ & 0.365 & & \\
\hline $\operatorname{Albumin}(\mathrm{g} / \mathrm{dL})$ & $\leqq 3$ vs. $>3$ & $2.028(0.872-4.716)$ & 0.101 & & \\
\hline Liver cirrhosis & Yes vs. No & $1.305(0.629-2.706)$ & 0.475 & & \\
\hline Child-Pugh grade & B vs. A & $1.735(0.526-5.723)$ & 0.365 & & \\
\hline ALBI grade & II/III vs. I & $3.126(1.415-6.916)$ & 0.005 & $3.689(1.512-8.997)$ & 0.004 \\
\hline Tumor size $(\mathrm{cm})$ & $>5$ vs. $\leqq 5$ & $2.242(1.163-4.321)$ & 0.016 & & \\
\hline Tumor no. & Multiple vs. Single & $3.705(1.133-12.113)$ & 0.03 & $9.993(2.177-45.866)$ & 0.003 \\
\hline BCLC stage & B vs. 0/A & $2.323(1.202-4.492)$ & 0.012 & & \\
\hline Liver fat content (\%) & $>30$ vs. $\leqq 30$ & $0.454(0.105-1.965)$ & 0.291 & & \\
\hline
\end{tabular}




\begin{tabular}{|c|c|c|c|c|c|}
\hline Vascular invasion & Yes vs. No & $0.618(0.290-1.317)$ & 0.213 & & \\
\hline pTNM stages & III vs. I+II & $6.395(2.171-18.834)$ & 0.001 & $8.853(2.795-28.043)$ & $<0.001$ \\
\hline Histology stages & Poor vs. well/moderate & $2.421(0.732-8.001)$ & 0.147 & & \\
\hline
\end{tabular}

$\mathrm{DM}=$ Diabetes mellitus; AST=Aspartate aminotransferase; ALBI = albumin-bilirubin; BCLC=Barcelona Clinic Liver Cancer; 
Figures

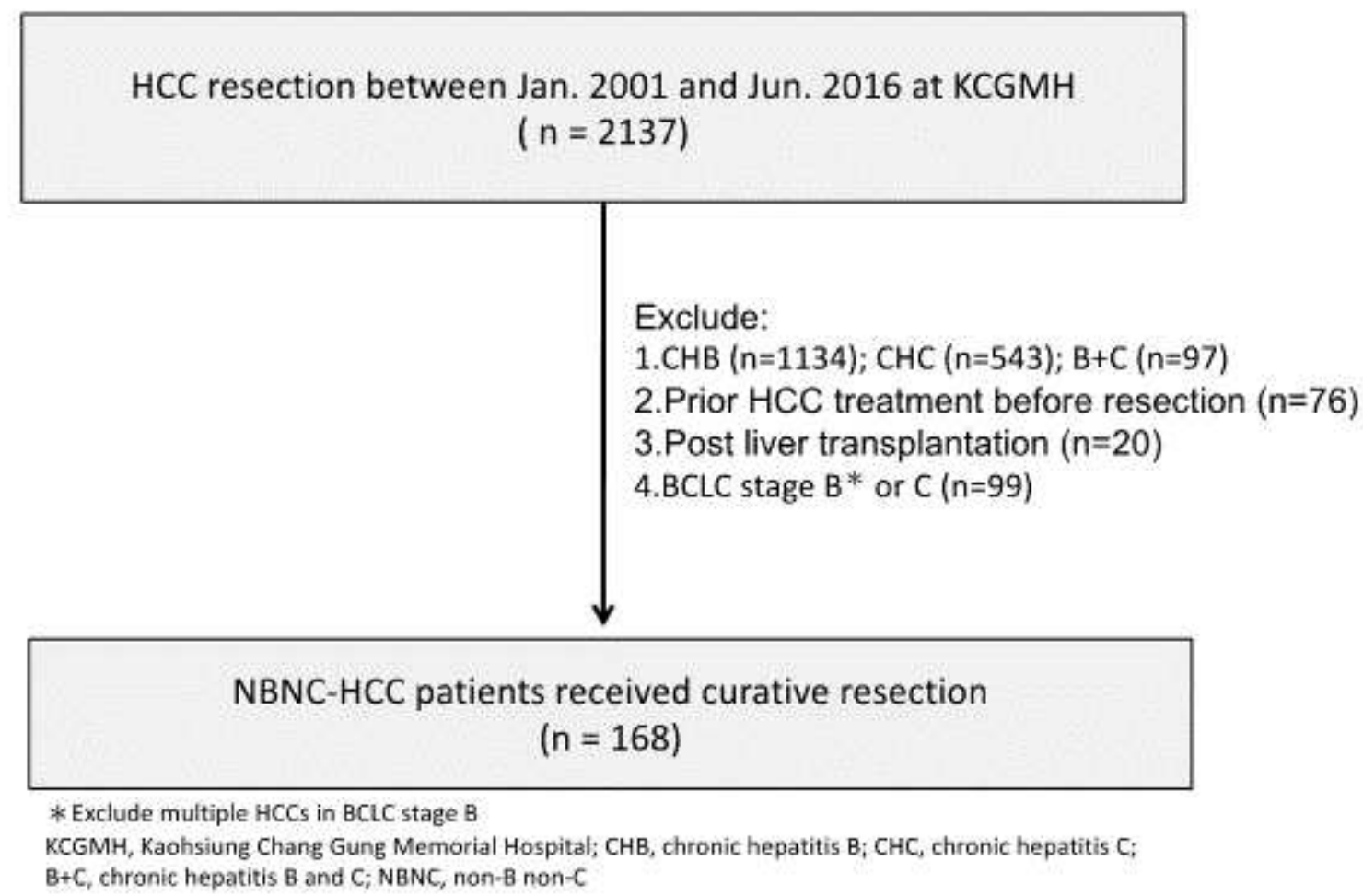

\section{Figure 1}

Schematic flowchart of the enrolment process 

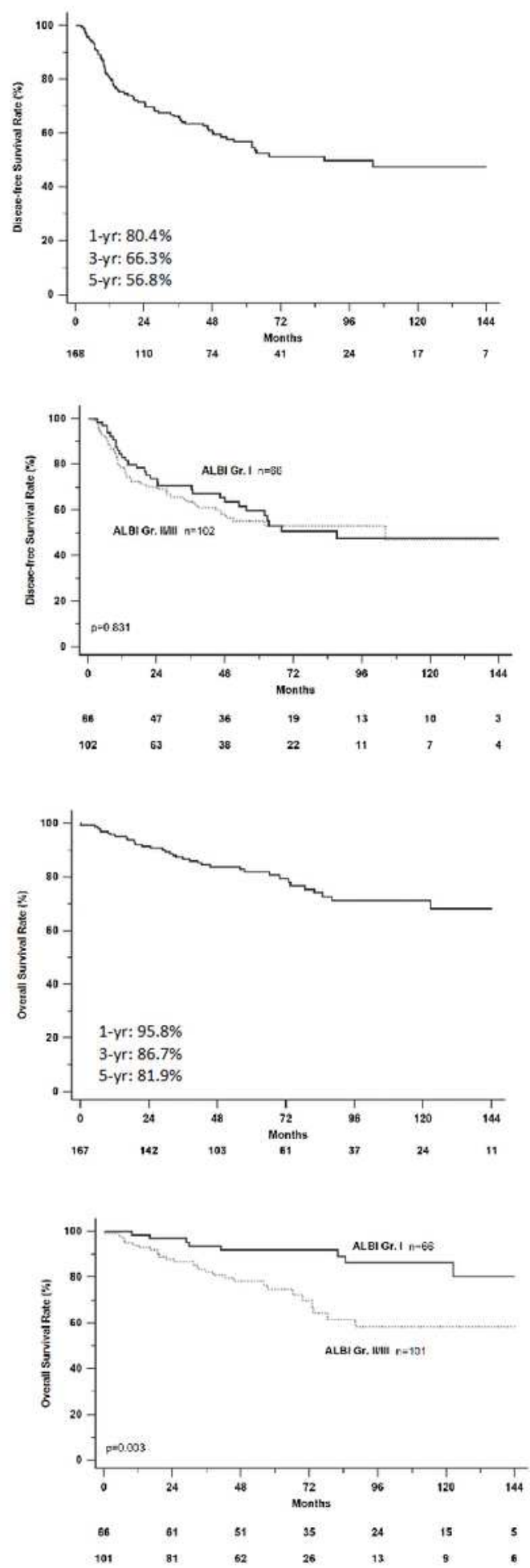

\section{Figure 2}

The disease-free survival (DFS) rate and overall survival (OS) rate in NBNC-HCC after resection. (A) DFS in the study population (B) DFS between ALBI Gr I vs. Gr II/III (C) OS in the study population (D) DFS between ALBI Gr I vs. Gr II/III. 

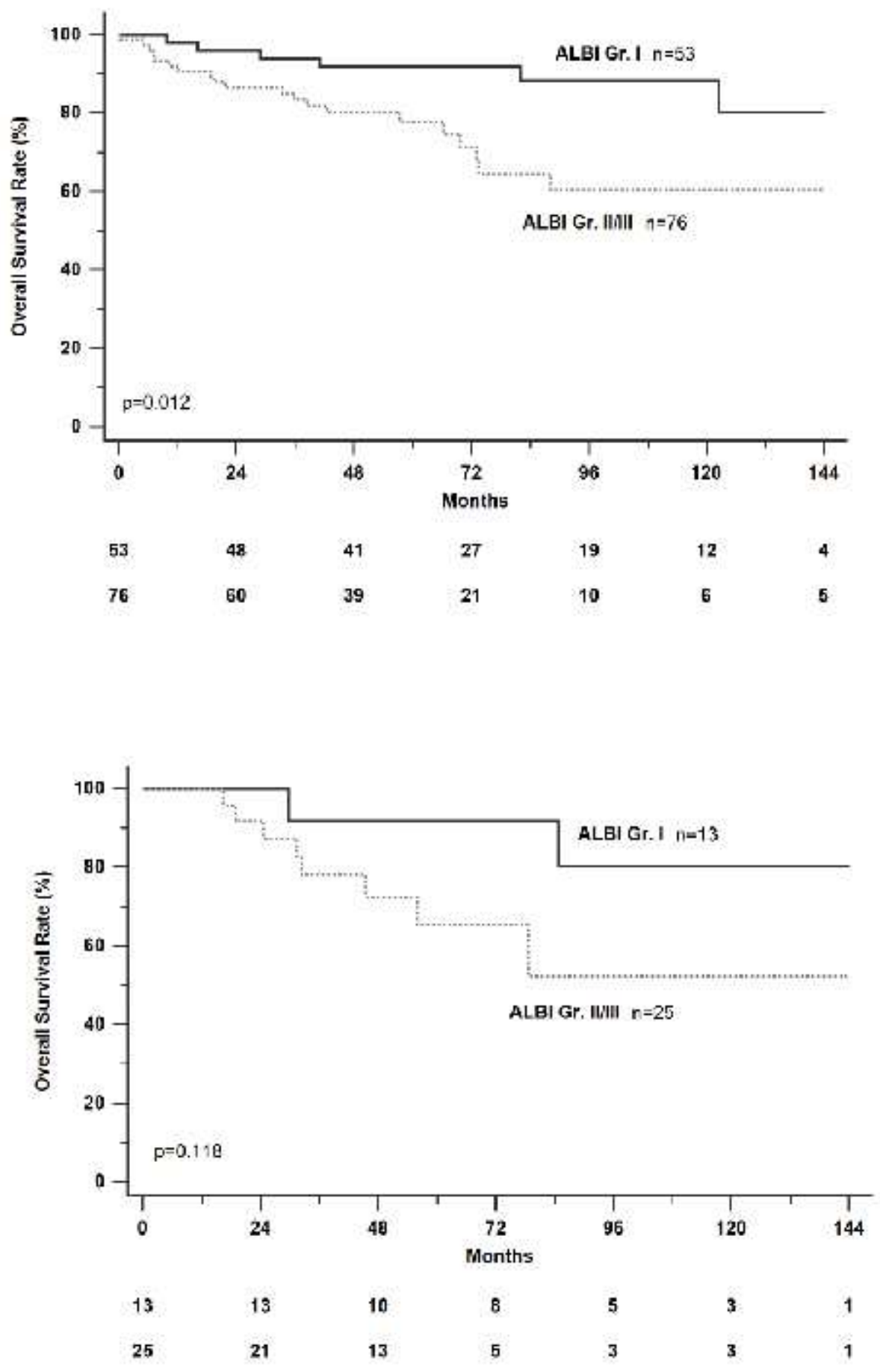

Figure 3

The cumulative overall survival in patients with NBNC-related HCC after curative resection among $(A)$ non cirrhotic patients and (B) cirrhotic patients. 